\title{
BENZILAÇÃO DO GLICEROL COM AQUECIMENTO POR MICROONDAS*
}

\author{
BENZYLATION OF GLYCEROL WITH HEATING BY MICROWAVE \\ BENZILACIÓN GLICERINA CON EL CALOR EN MICROONDAS
}

\author{
Mitchell González Soares da Silva \\ Kiany Sirley Brandão Cavalcante \\ Ulisses Magalhães Nascimento \\ Antônio Carlos Sales Vasconcelos \\ Adeilton Pereira Maciel \\ Fernando Carvalho Silva
}

\begin{abstract}
Resumo: A infinidade de aplicações da glicerina incentivou inúmeras pesquisas voltadas ao reaproveitamento do principal coproduto do biodiesel. Destaca-se a produção de aditivos oxigenados para combustíveis, como os éteres, os acetais e os ésteres de glicerina, processos estes que demandam um tempo de reação muito prolongado. Considerando que a aplicação da irradiação de microondas pode influenciar na cinética das reações químicas, o presente trabalho investigou a reação de benzilação do glicerol via catálise homogênea ácida, sob a irradiação de microondas. Foi constatado que o fator variação da potência das micro-ondas apresentou a maior conversão do glicerol dentre todas as variáveis estudadas. Em contrapartida, esta variável também influenciou uma reação indesejável, a autoeterificação do álcool benzílico.
\end{abstract}

Palavras-chave: Benzilação. Glicerol. Álcool benzílico. Microondas.

\begin{abstract}
The multitude of applications of glycerin has encouraged countless researches directed to the reutilization of the main byproduct of biodiesel. Highlighting the production of additives for oxygenated fuels, such as the ethers, the acetal and the esters of glycerin, those processes require a very long reaction time. Whereas the application of irradiation of microwave can influence on the kinetics of chemical reactions, the present work investigated the benzylation reaction of glycerol via catalysis homogeneous acid, in the irradiation of microwave. It was found that the factor power variation of microwave presented the greatest conversion of glycerol among all the variables studied. In contrast, that variable also influenced an undesirable reaction, the self-etherification of benzyl alcohol.
\end{abstract}

Keywords: Benzylation. Glycerol. Benzyl alcohol. Microwave.

Resumen: La grande cantidad de aplicaciones de la glicerina ha alentado numerosos estudios dirigidos a la reutilización del principal subproducto de biodiesel. Destacando la producción de aditivos oxigenados de combustibles para motores, tales como éteres, acetales y ésteres de glicerina, estos procedimientos requieren un tiempo de reacción muy largo. Considerando que la aplicación de irradiación de microondas puede influir en la cinética de las reacciones químicas, este estudio investigó la reacción de bencilación de glicerol a través de catálisis homogénea ácida bajo irradiación con microondas. Se encontró que la variación del factor potencia de microondas mostró la mayor conversión de glicerol entre todas las variables evaluadas. En contraste, esta variable también influenció una reacción indeseable, la auto-eterificación de alcohol bencílico.

Palabras clave: Bencilación. Glicerol. Alcohol bencílico. Microondas.

\section{INTRODUÇÃO}

Em consequência dos problemas ambientais causados pelo uso do petróleo, inúmeros países adotaram estratégias políticas baseadas em fontes de energias renováveis, como a energia fotovoltaica, eólica e biomassa, nesta destacando-se o biodiesel (CAVALCANTE et al., 2012, p. 5).

No Brasil, o crescimento da produção do biodiesel, incentivado pelo governo federal, com a adição de $5 \%$ de biodiesel ao diesel (CONSELHO NACIONAL DE POLÍTCA ENERGÉ
TICA, 2009, p.1), despertou a preocupação para destino da glicerina bruta, subproduto da transesterificação de triacilglicerídeos. Dados da ANP estimam que as usinas brasileiras atualmente disponibilizem no mercado interno um volume de quase $2 \mathrm{mil} \mathrm{m}^{3}$. dia-1 de glicerina bruta (AGÊNCIA NACIONAL DO PETRÓLEO, GÁS NATURAL E BIOCOMBUSTÍVEIS, 2011), deste modo estão sendo investigados novos processos para o aproveitamento deste resíduo.

\footnotetext{
*Artigo recebido em setembro 2012
}

Aprovado em dezembro 2012 
Estudos revelam que a glicerina pode ser convertida a aditivos oxigenados para combustíveis, como éteres (MELERO et al., 2008, p.44), acetais (MOTA, 2009, p.38) e ésteres de glicerina (BALARAJU et al., 2010, p.249). Em contrapartida, as reações químicas para obtenção desses compostos, que geralmente utilizam o aquecimento por condução térmica, requerem tempos de reação prolongados (KARINEN; KRAUSE, 2003, p.129). Alternativamente ao aquecimento convencional, a tecnologia de irradiação de micro-ondas permite um aumento na velocidade das reações em reações orgânicas (NASCIMENTO et al., 2009, p.37).

A reação de benzilação do glicerol utilizando aquecimento por condução térmica requer um tempo de reação prolongado. Esse período pode ser reduzido usando aquecimento por micro-ondas, que incide geralmente por rotação de dipolo ou condução iônica. Nessa perspectiva, o presente trabalho tem o objetivo de estudar a conversão da glicerina em éteres benzílicos de glicerol, usando o aquecimento por irradiação de micro-ondas.

\section{METODOLOGIA}

\subsection{Benzilação do glicerol por microondas}

As reações de eterificação do glicerol com álcool benzílico foram realizadas em um forno de microondas doméstico (35 litros, com gerador de micro-ondas na faixa de frequência de $2.450 \mathrm{MHz}$ ), adaptado para reações químicas com um balão de vidro, um condensador de alta eficiência, um termopar conectado a um controlador de temperatura e um adaptador eletrônico de acionamento das teclas liga e desliga (NASCIMENTO et al., 2009, p.38).

Incialmente as reações com aquecimento por micro-ondas foram comparadas com reações que aplicam aquecimento convencional utilizando catálise homogênea e heterogênea. As condições reacionais usadas consistem em: razão molar glicerina:álcool benzílico 1:3, $110{ }^{\circ} \mathrm{C}$ e $1 \%$ de catalisador ácido homogêneo (ácido p-toluenosulfônico, $\mathrm{TsOH}$ ) ou heterogêneo (Amberlyst 15, A-15). As reações foram realizadas em 3 horas com aquecimento por condução térmica e 1 hora por micro-ondas. O procedimento experimental consiste na solubilização do catalisador TsOH no álcool benzílico previamente calculado em razão molar glicerol:álcool, e depois a mistura é transferida para balão de reação, contendo o glicerol.
As variáveis operacionais que influenciam na conversão do glicerol foram avaliadas em função do tempo (25, 50, 75 e 100 minutos): temperatura $(T)$, potência do microondas $(P)$, catalisador (C) e razão molar glicerol:álcool (RM), conforme demonstra a Tabela 1.

Tabela 1 - Influência das variáveis ( $T, P, C$ e RM) em função do tempo de reação $(25,50,75$ e 100 minutos)

\begin{tabular}{c|c|c|c}
\hline $\begin{array}{c}\text { Razão } \\
\text { Molar }\end{array}$ & $\begin{array}{c}\text { Catalisador } \\
(\%)\end{array}$ & $\begin{array}{c}\text { Temperatura } \\
\left({ }^{\circ} \mathbf{C}\right)\end{array}$ & Potência \\
\hline \multicolumn{4}{c}{ Influência da temperatura (T) } \\
\hline $1: 3$ & 1,0 & 90 & 5 \\
\hline \multicolumn{4}{c}{ Influência da potência (P) } \\
\hline $1: 3$ & 1,0 & 110 & 10 \\
\hline \multicolumn{4}{c}{ Influência do catalisador (C) } \\
\hline $1: 3$ & 1,5 & 110 \\
\hline \multicolumn{4}{c}{ Influência da razão molar (RM) } \\
\hline $1: 4$ & 1,0 & 110 & 5 \\
\hline
\end{tabular}

Fonte: Elaborada pelos autores

\subsection{Caracterização do produto da reação}

A caracterização dos produtos formados foi realizada usando as técnicas de Espectroscopia na Região do Infravermelho com Transformada de Fourier (FTIR), Cromatografia de Camada Delgada (CCD) e Cromatografia a Gás com detector por Ionização em Chama (CG-DIC). A técnica de FTIR foi realizada utilizando um espectrômetro da Shimadzu, IRPrestige-21, na região entre 4000 e $400 \mathrm{~cm}^{-1}$ e pastilhas de brometo de potássio. A CCD empregou uma placa cromatográfica Macherey-Nagel com 0,20 mm de sílica gel 60 suportada em alumina e como eluente a mistura haxano:éter etílico:ácido acético 8:2:0,2. Na CG-DIC utilizou-se um Cromatógrafo Shimadzu GC-2010, uma coluna capilar de sílica fundida Shimadzu (5 \% fenil e $95 \%$ PDMS, $30 \mathrm{~m} \times 0,32 \mathrm{~mm} \times 0,25 \mu \mathrm{m})$ e as seguintes condições: $250^{\circ} \mathrm{C}$ de temperatura no detector; $250^{\circ} \mathrm{C}$ de temperatura no injetor; programação de temperatura no forno de $50^{\circ} \mathrm{C}$ por $5 \mathrm{~min}$, de 50 a $180^{\circ} \mathrm{C}\left(10^{\circ} \mathrm{C} / \mathrm{min}\right), 180^{\circ} \mathrm{C}$ por $2 \mathrm{~min}$ e de 180 a $230^{\circ} \mathrm{C}\left(5^{\circ} \mathrm{C} / \mathrm{min}\right)$ e $230^{\circ} \mathrm{C}$ por $4 \mathrm{~min}$. Foram injetados $1 \mu \mathrm{L}$ da amostra diluída em 2-propanol. Os padrões cromatográficos comerciais 3-benzióxi-1,2-propanodiol (Fluka, $\geq 97 \%$ ), éter dibenzílico (Fluka, $\geq 98 \%$ ) e glicerol (Cromoline, $99,5 \%$ ) foram usados para confirmar os tempos de retenção nos cromatogramas das amostras, quantificando-os por meio do método de Calibração de Multicomponentes, com base em medições de calibração (JAMRÓZ et al., 2007). 


\section{RESULTADOS E DISCUSSÃO}

\subsection{Influência das microondas na benzi- lação do glicerol}

$\mathrm{Na}$ análise qualitativa do produto da reação de benzilação do glicerol por $C C D$, demonstrou-se nas cromatoplacas de sílica, reveladas com iodo, uma conversão significativa da glicerina em éteres, visto a presença de uma mancha acentuada com poucos vestígios do material de partida, o glicerol. Nas reações catalisadas com A-15, foi possível detectar o início de conversão da glicerina em menor tempo, 7 minutos, enquanto aquelas catalisadas com $\mathrm{TsOH}$ necessitaram de 15 minutos, que por sua vez é inferior ao tempo necessário para reações com aquecimento convencional, 3 horas (Figura 1 ).

De fato, esperava-se esse acontecimento, pois o efeito que as micro-ondas ocasionam é baseado na polarização das moléculas e dos íons livres de materiais dielétricos, induzida pelo campo elétrico das radiações (FORTUNY et al., 2008, p.1554), e a glicerina e o álcool benzílico são substâncias polares.

Figura 1 - CCD dos produtos de reação de eterificação da glicerina

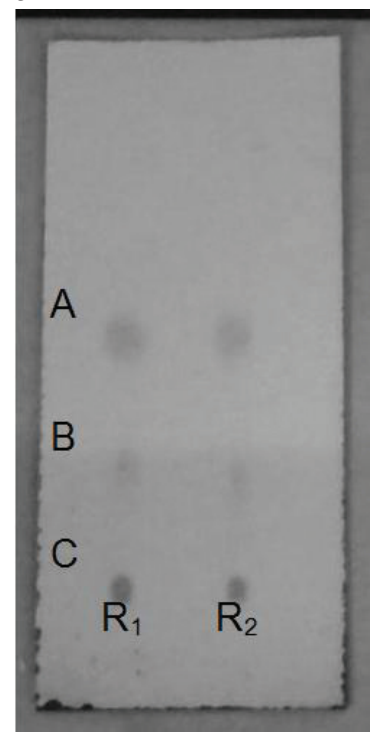

*Legenda: R1: aquecimento convencional e $\mathrm{TsOH}$; R2: aquecimento por micro-ondas e $\mathrm{TsOH}$

A e B: Produtos eterificados; C: Glicerina Fonte: Elaborada pelos autores

O catalisador heterogêneo A-15 apresentou conversão em éteres de glicerol em tempo menor que o $\mathrm{TsOH}$, mostrando grande potencial para esta reação, no entanto a resina de troca iônica apresentou um inchamento das esferas ao longo da reação até a sua total des- fragmentação, devido possivelmente à contínua formação dos éteres de glicerol e éter dibenzílico e/ou da água, subproduto da reação de eterificação. Portanto, avaliou-se o estudo das variáveis das reações com o catalisador homogêneo.

O produto da reação também foi analisado por meio de espectros na região do infravermelho médio $\left(4000-400 \mathrm{~cm}^{-1}\right)$ que revelaram modos vibracionais característicos dos éteres de glicerina (Gráfico 1), com base nas atribuições espectrais dos respectivos grupos constituintes.

Gráfico 1 - Espectros de infravermelho do produto da reação de eterificação da glicerina

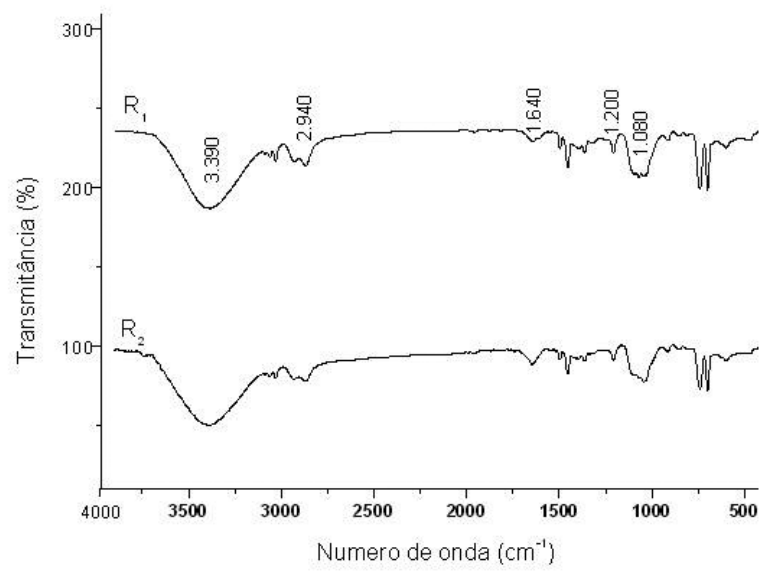

Legenda: $\mathrm{R}_{1}$ : aquecimento convencional e $\mathrm{TsOH}$; R2: aquecimento por micro-ondas e $\mathrm{TsOH}$ Fonte: Elaborada pelos autores

O espectro vibracional na região do IV das reações apresentou bandas de absorção intensas com modos vibracionais em 3.390 $\mathrm{cm}^{-1}$, atribuídas às vibrações de estiramento e flexão dos grupos hidroxila, provenientes possivelmente dos éteres de glicerina mono- e di- substituídos e da glicerina residual. Os modos vibracionais referentes aos estiramentos das ligações $\mathrm{C}-\mathrm{H}, \mathrm{C}=\mathrm{C}, \mathrm{C}-\mathrm{O}$ e $\mathrm{C}-\mathrm{O}-\mathrm{C}$, característica de éteres, foram localizados nas regiões de 2.940, 1.640, 1.200 e $1.080 \mathrm{~cm}^{-1}$, respectivamente.

A conversão do glicerol induzida por micro-ondas ao longo do tempo de reação foi ponderada devido a estudos que apontam os efeitos cinéticos de irradiações por microondas em reações químicas (WANG et al., 2003, p.933; ZOU et al., 2009, p.17). As taxas de conversão do glicerol, assim como da produção do mono-éter (3-benzióxi-1,2propanodiol) e éter dibenzílico ao longo do tempo estão apresentadas no Gráfico 2. 
Gráfico 2 - Percentual da conversão glicerol (a) e produção do mono-éter (b) e éter dibenzílico (c), obtidas durante o estudo das variáveis ( $T, P, C$ e RM) em função do tempo de reação $(25,50,75$ e 100 minutos)

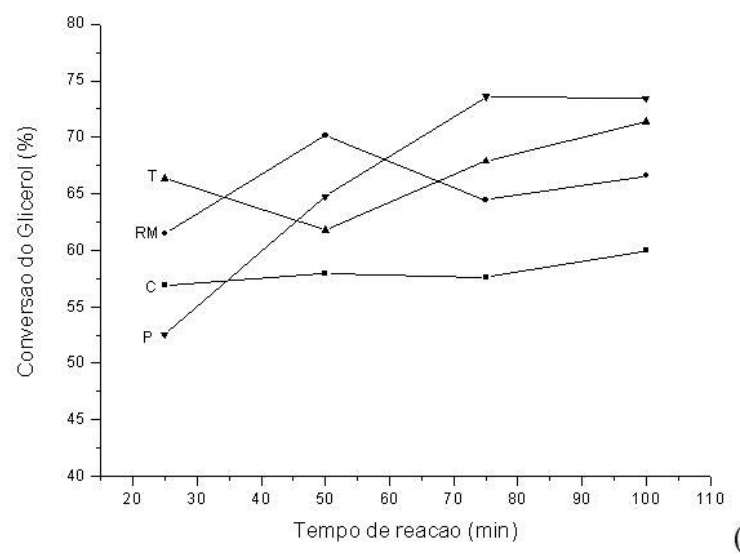

(a)

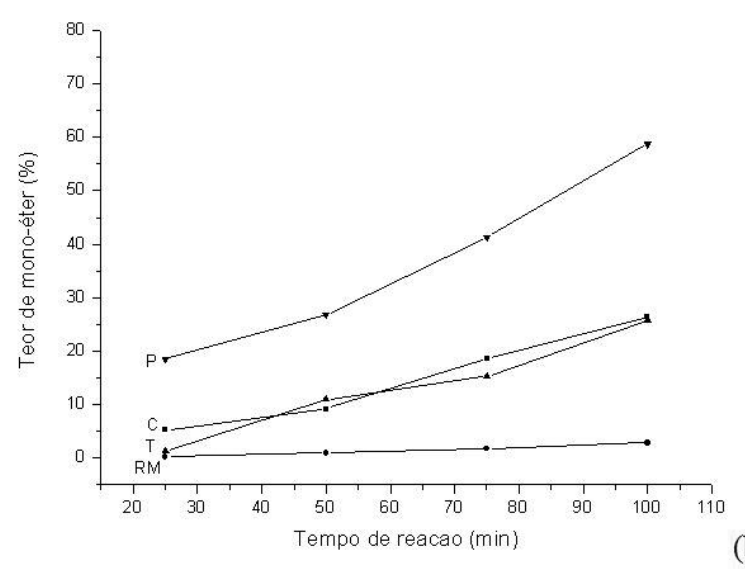

(b)

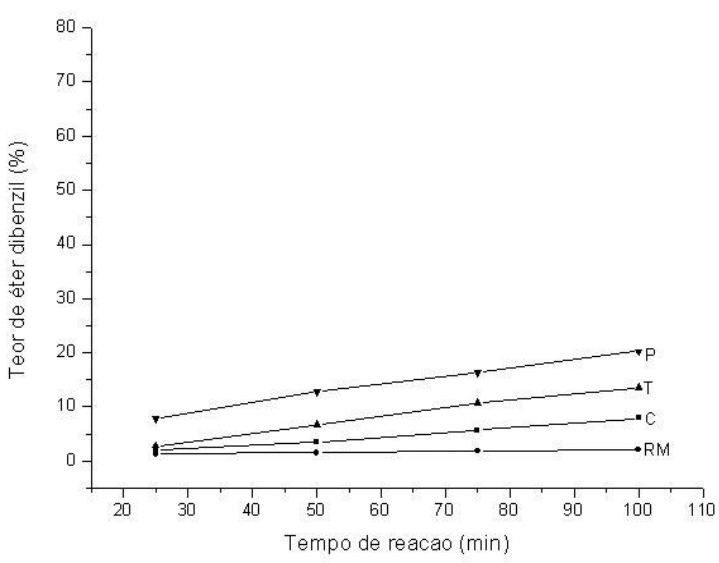

(c)

Legenda: T: Temperatura; P: Potência; C: Catalisador; RM: Razão molar.

Fonte: Elaborada pelos autores

A potência das irradiações por micro-ondas foi o fator que mais variou ao longo do tempo, com um crescente consumo do glicerol e, consequentente, produção do mono-éter de glicerol em aproximadamente 80 minutos, abaixo do tempo necessário para conversão usando aquecimento convencional (120 minutos). En- tretanto, acarretou um aumento da autoeterificação do álcool benzílico, formando o éter dibenzílico, reação secundária.

A temperatura apresentou um pico de conversão máximo em 100 min. Considerando-se que as reações aquecidas com micro-ondas ocorrem da interação matéria/campo elétrico da irradiação incidente, capaz de produzir calor e, consequentemente, aumentar a temperatura do meio (FORTUNY et al., 2008, p. 1553), justifica o aumento da temperatura do sistema com 75 minutos de reação e, consequentemente, uma maior conversão do glicerol.

Isso ocorre, porque geralmente o tempo de relaxação de um material aumenta com a temperatura, aumentando a potência de micro-ondas absorvida (CARVALHO, 2005, p.27).

Com um excesso do agente eterificante esperava-se provocar um deslocamento do equilíbrio no sentido de maior consumo do glicerol, entretanto com 50 minutos houve uma redução na conversão do glicerol, devido ao aumento da autoeterificação do álcool que disputa simultaneamente com a reação desejada.

\subsection{Linearidade na análise cromatográfi- ca CG-DIC}

As reações realizadas no estudo da conversão da glicerina em função do tempo foram acompanhadas pela análise cromatográfica CG-DIC. O método cromatográfico usado foi capaz de distinguir os componentes da mistura: 2-propanol, éter dibenzílico, mono-éter, glicerol e supostamente o di-éter (2,3-di-benzióxi-propan-1-ol) ou tri-éter (propano 1,2,3-tri-benzil-butoxi) produto da benzilação do mono-éter, conforme o Gráfico 3.

Gráfico 3 - Cromatograma do produto de reação de benzilação do glicerol

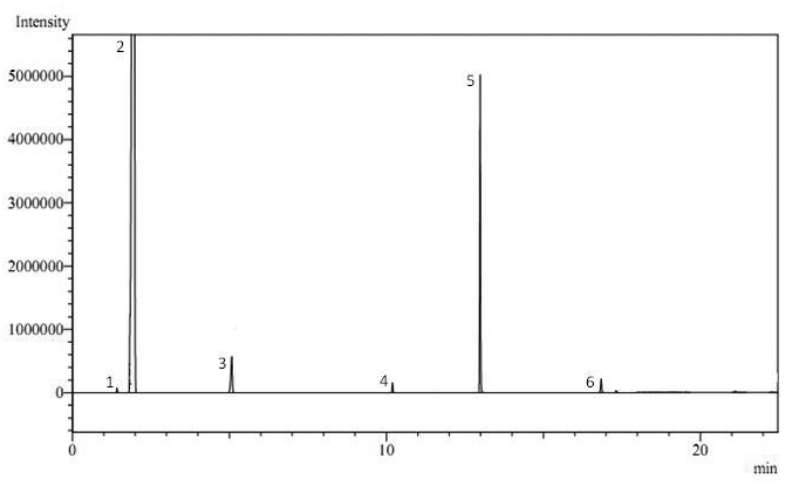

Legenda: 1: éter dibenzílico; 2: 2-pronanol; 3 e 4: di-éter ou tri-éter; 5: mono-éter; 6: glicerol

Fonte: Elaborada pelos autores 
O intervalo de linearidade, que mede quanto uma curva de calibração segue uma linha reta, foi determinado a partir das curvas analíticas apenas para três analitos (glicerol, mono-éter e éter-dibenzílico), pois não existem comercialmente na forma de padrões cromatográficos os di- e tri-éteres. O Gráfico 4 apresenta as curvas analíticas e as suas respectivas retas de regressão linear simples, representadas pela equação $y=a+b x$, sendo y $a$ área do pico e $x$ a concentração do analito.

Gráfico 4 - Curva de calibração para o glicerol (a), mono-éter (b) e éter dibenzílico (c)

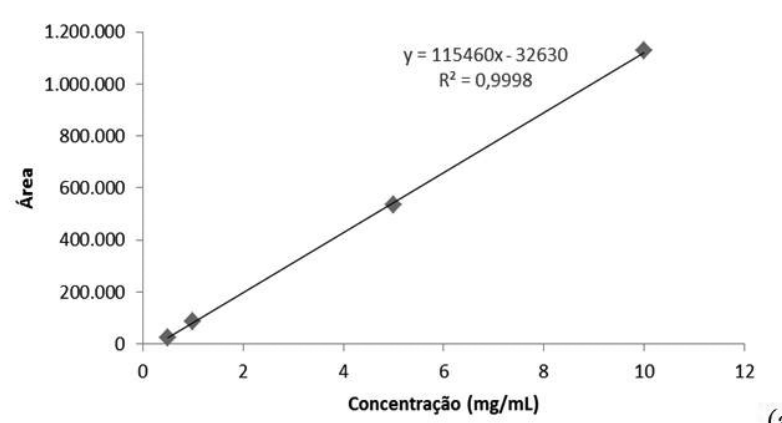

(a)

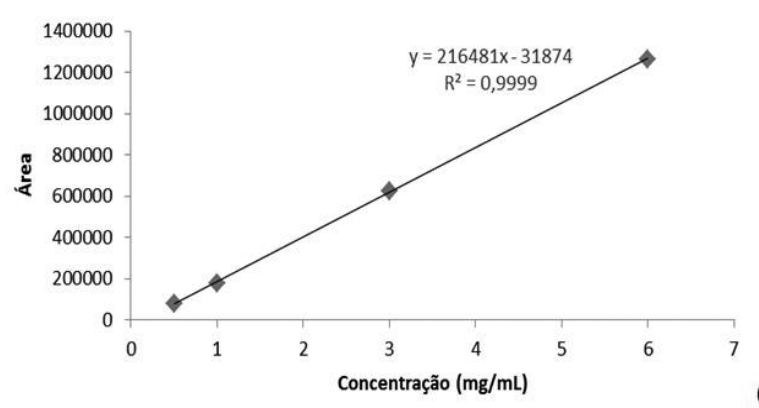

(b)

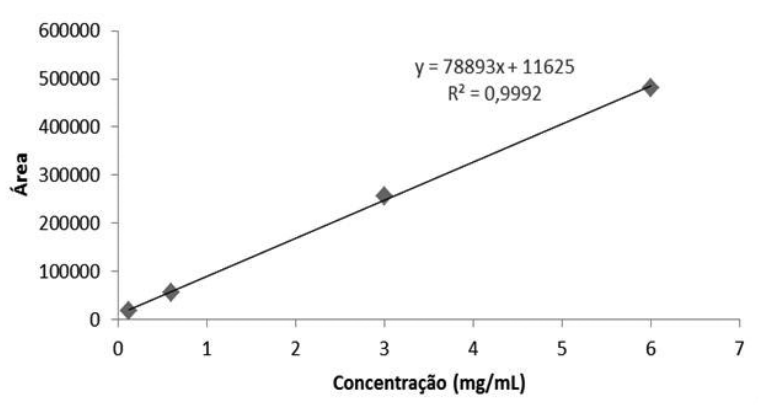

(c)

Fonte: Elaborada pelos autores

Uma análise com base na inspeção dos gráficos obtidos e nos valores do $\mathrm{R}^{2}$, que mede a qualidade de confiança depositada na equação de regressão como instrumento de precisão (HARRIS, 2008, p.93) para o intervalo contendo os quatro pontos, indicaram a linearidade das curvas de calibração, ou seja, apresentaram valores acima de 0,999, o que corresponde a um excelente ajuste linear.

\section{CONIDERAÇÕES FINAIS}

Admite-se que o processo de benzilação da glicerina com aquecimento por micro-ondas é uma alternativa promissora. As reações via catálise homogênea ácida induzida por micro-ondas apresentaram uma conversão do glicerol em menor tempo, comparado ao processo com aquecimento por condução térmica. A potência das micro-ondas foi o fator que mais variou ao longo do tempo com uma crescente produção do mono-éter de glicerol. Em contrapartida, ela também influenciou uma reação indesejável, a autoeterificação do álcool benzílico, sendo, assim, necessário um estudo de otimização do processo para a produção dos éteres de glicerol.

\section{AGRADECIMENTOS}

M.G.S. da S. agradece ao CNPq pela bolsa PIBIC. Os autores agradecem aos órgãos de fomento à pesquisa FINEP, CNPq e FAPEMA pelo apoio financeiro ao Núcleo de Biodiesel da UFMA.

\section{REFERÊNCIA}

AGÊNCIA NACIONAL DO PETRÓLEO, GÁS NATURAL E BIOCOMBUSTÍVEIS. Capacidade autorizadas das plantas de produção de biodiesel. Disponível em: <http://www.anp. gov.br/>. Acesso em: 6 set. 2011.

BALARAJU, M.; et al. Acetylation of glycerol to synthesize bioadditives over niobic acid supported tungstophosphoric acid catalysts. Fuel Process. Technol., v. 91, p. 249, 2010.

CARVALHO, R. P. de. Microondas. São Paulo: Ed. Livraria Física; SBF, 2005. (Coleção temas atuais de física).

CAVALCANTE, K. S. B. et al. Oxygenated glycerol derivatives as an alternative source of energy: a review. Eclet. Quím., v. 36, n. 4 , p. 5-14, 2012.

CONSELHO NACIONAL DE POLÍTICA

ENERGÉTICA. Resolução no 6, de 16.9.2009. Diário Oficial da União, Brasília, DF, 26 out. 2009.

FORTUNY, M. et al. Principais aplicações das microondas na produção e refino de petróleo. Quim. Nova, v. 31, n. 6, p. 1553, 2008.

HARRIS, D. C. Análise química quantitativa. 3.ed. Rio de Janeiro: LTC, 2008. 
KARINEN, R. S.; KRAUSE, A. O. I. New biocomponents from glycerol. Appl. Catal. A., v. 306, p. 128, 2003.

MELERO, J. A. et al. Acid-catalyzed etherification of bio-glycerol and isobutylene over sulfonic mesostructured silicas. Appl.

Catal. A: Gen., v. 346, p. 44, 2008.

MOTA, C J. A.; GONÇALVES, V. L. C.; SILVA, C. X. A. da. Water-tolerant zeolite catalyst for the acetalisation of glycerol. Green Chem., v. 11, p. 38, 2009.
NASCIMENTO, U. M. et al. Otimização da produção de biodiesel a partir de óleo de coco babaçu com aquecimento por microondas. Eclet. Quím., v. 34, n. 4, p. 37, 2009.

WANG, W. G. et al. Emissions from nine heavy trucks fueled by diesel and biodiesel blend without engine modification. Environ. Sci. and Technol., v. 34, n. 6, p. 933, 2000. ZOU, J.; LI, F.; TAO, F. G. Microwaveassisted synthesis of diaryl or aryl-alkyl sulfones without catalyst. Chinese Chem. Lett., v. 20, p. 17, 2009. 